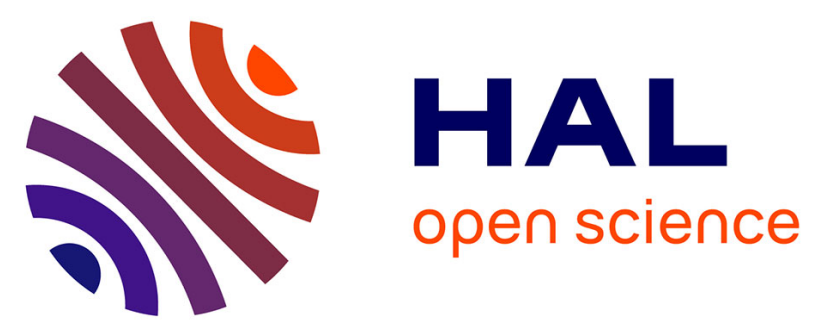

\title{
Measurements of Diffusion and Perfusion in Vertebral Bone Marrow Using Intravoxel Incoherent Motion (IVIM) With Multishot, Readout-Segmented (RESOLVE) Echo-Planar Imaging
}

Jérémy Lasbleiz, Caroline Le Ster, Raphaël Guillin, Hervé Saint-Jalmes, Giulio Gambarota

\section{To cite this version:}

Jérémy Lasbleiz, Caroline Le Ster, Raphaël Guillin, Hervé Saint-Jalmes, Giulio Gambarota. Measurements of Diffusion and Perfusion in Vertebral Bone Marrow Using Intravoxel Incoherent Motion (IVIM) With Multishot, Readout-Segmented (RESOLVE) Echo-Planar Imaging. Journal of Magnetic Resonance Imaging, 2019, 49 (3), pp.768-776. 10.1002/jmri.26270 . hal-01880047

\section{HAL Id: hal-01880047}

\section{https://hal-univ-rennes1.archives-ouvertes.fr/hal-01880047}

Submitted on 28 Sep 2018

HAL is a multi-disciplinary open access archive for the deposit and dissemination of scientific research documents, whether they are published or not. The documents may come from teaching and research institutions in France or abroad, or from public or private research centers.
L'archive ouverte pluridisciplinaire HAL, est destinée au dépôt et à la diffusion de documents scientifiques de niveau recherche, publiés ou non, émanant des établissements d'enseignement et de recherche français ou étrangers, des laboratoires publics ou privés. 
Measurements of diffusion and perfusion in vertebral bone marrow using intravoxel incoherent motion (IVIM) with multi-shot, readout-segmented (RESOLVE) echo-planar MRI

Jérémy Lasbleiz, MD, $P h D^{1}$, Caroline Le Ster, $P h D^{1,2}$, Raphaël Guillin, $M D^{4}$,Hervé Saint-Jalmes, $P h D^{3}$ and Giulio Gambarota, $P h D^{1}$

1 Univ Rennes, Inserm, LTSI - UMR 1099, F-35000 Rennes, France 2 Siemens Healthcare, Saint-Denis, France

3 Univ Rennes, CLCC Eugène Marquis, Inserm, LTSI - UMR 1099, F-35000 Rennes, France 4 Department of Medical Imaging, Rennes University Hospitals, Sud Hospital, F-35000 Rennes, France 


\section{ABSTRACT}

BACKGROUND: A limited number of studies have used the intravoxel incoherent motion (IVIM) approach on bone marrow. In none of the previous studies, the effects of fat suppression on the IVIM parameters have been investigated.

PURPOSE: To measure the water diffusion coefficient and the perfusion fraction in vertebral bone marrow using IVIM with multi-shot, readout-segmented (RESOLVE) echo-planar imaging and to assess the effects of different fat suppression techniques on the measurement of the IVIM parameters.

STUDY TYPE: Prospective.

POPULATION/SUBJECTS: Six healthy volunteers (24.2 \pm 4.3 years).

FIELD STRENGTH/SEQUENCE: 1.5T, RESOLVE.

ASSESSMENT: Four experiments were performed: 1) RESOLVE imaging without fat suppression, 2) with fat saturation (FS), 3) with SPAIR and 4) with STIR. The water diffusion coefficient $D$, pseudo-diffusion coefficient $D^{*}$ and the perfusion fraction $f$ were assessed in the vertebral bodies of the lumbar vertebrae.

STATISTICAL TESTS: One-way repeated-measures analysis of variance (ANOVA) followed by Bonferroni's multiple comparison test.

RESULTS: The RESOLVE IVIM protocol allowed for measurement of $D, D^{*}$ and $f$ in all volunteers. The signal of lipid protons affected the quantification of the IVIM diffusion coefficient: $D=0.24 \pm 0.10\left(\times 10^{-3} \mathrm{~mm}^{2} / \mathrm{s}\right)$, no fat suppression; $D=0.43 \pm 0.07$ $\left(x 10^{-3} \mathrm{~mm}^{2} / \mathrm{s}\right), \mathrm{FS} ; \mathrm{D}=0.42 \pm 0.07\left(\mathrm{x} 10^{-3} \mathrm{~mm}^{2} / \mathrm{s}\right)$, SPAIR; $\mathrm{D}=0.35 \pm 0.10\left(\times 10^{-3} \mathrm{~mm}^{2} / \mathrm{s}\right)$ STIR; and IVIM perfusion fraction $f=7.5 \pm 1.9 \%$ no fat suppression; $f=14.5 \pm 5.4 \%$, FS; $f=12.5 \pm 2.6 \%$, SPAIR; $f=18.1 \pm 6.1 \%$, STIR. No significant effect $(p=0.36)$ was found on the quantification of $D^{*}$. 
DATA CONCLUSION: An IVIM-MRI protocol using the RESOLVE sequence was implemented for measurements of vertebral bone marrow diffusion and the perfusion. The comparison between the protocols with and without fat suppression indicates that the lipid signal results in an underestimation of both $D$ and $f$. 


\section{INTRODUCTION}

Intravoxel incoherent motion (IVIM) MRI is a technique that allows for simultaneous assessment of the water diffusion coefficient $(D)$ and perfusion fraction $(f)(1)$. The sensitization of the MR signal to IVIM is accomplished by acquiring MR images with diffusion-sensitizing gradients strengths (b-values). Fitting the IVIM signal decay to a biexponential function allows for determining both $D$ and $f$. Furthermore, the biexponential fit yields also the pseudo-diffusion coefficient $D^{*}$, although the estimation of this parameter notoriously suffers from greater uncertainty (2).

The quality of the MR diffusion-weighted images has substantially improved over the last two decades. As a result, the IVIM technique can now be applied to various tissues. Surprisingly, however, only a very limited number of IVIM studies have been performed on bone marrow (3-9). This might be due to lipids which represent a major confounding factor in the quantification of both diffusion and perfusion $(10,11)$. While the effects of the lipids on the quantification of the water (apparent) diffusion coefficient $(A D C)$ in bone marrow have been investigated (12), little attention has been paid to their effects on the diffusion $\left(D, D^{*}\right)$ and perfusion $(f)$ parameters obtained by the IVIM approach. Furthermore, since fat suppression techniques are mandatory for an accurate quantification of $D, D^{*}$ and $f$, it is important to test the effects of the different techniques available on clinical MR systems.

Furthermore, it is also noteworthy that IVIM studies on bone marrow have been performed over the last three years and with single-shot EPI (Echo-Planar Imaging) sequences. In recent years, a new diffusion sequence (RESOLVE, read-out segmentation of Iong variable echo train) has been introduced clinically (13). This sequence allows for multi-shot readout-segmented EPI acquisitions of the k-space. The advantages of RESOLVE over single-shot EPI include the acquisition of MR 
images with an increased spatial resolution and reduced distortions, at the cost however of longer acquisition times.

The RESOLVE sequence has found many applications; in particular, it has been suggested that this MRI sequence is of interest for spine imaging (14), where susceptibility and blurring artifacts typically degrade image quality and have a negative impact on the assessment of quantitative diffusion parameters. Previous studies using the RESOLVE sequence have focused on diffusion-weighted and diffusion tensor imaging (13-16).

The aim of this study was twofold: first, to quantify diffusion and perfusion in the vertebral bone marrow with IVIM, using the RESOLVE sequence; second, to investigate the effects of the lipid signal and the different fat suppression techniques on the quantification of the IVIM parameters. 


\section{MATERIALS AND METHODS}

\section{MR Imaging}

All experiments were conducted according to the procedures approved by the local Institutional Review Board. Written informed consent was obtained from all participants. MRI acquisitions were performed on a $1.5 \mathrm{~T}$ MR system MAGNETOM Aera (Siemens Healthcare, Erlangen, Germany) using spine and body receiver coils. MRI acquisitions were carried out on the lumbar spine of six healthy volunteers (age range 20 to 32 years, mean age $24.2 \pm 4.3$ years, 3 women and 3 men). One volunteer (a 25-year-old man) was scanned six times to perform a repeatability study. Four IVIM acquisitions were performed in sagittal orientation using the RESOLVE sequence. One MRI acquisition was carried out without fat suppression; in each of the other three RESOLVE acquisitions, one of the following fat suppression techniques provided by the system vendor was used: Fat Saturation (FS), Spectral Attenuated Inversion Recovery (SPAIR) and Short-Tau Inversion Recovery (STIR). The sequence parameters of the spine IVIM protocol are provided in Table 1. Parallel imaging with GRAPPA (GeneRalized Autocalibrating Partially Parallel Acquisitions) was employed. Furthermore, in each volunteer a multi-echo VIBE-DIXON acquisition was performed, in order to determine the fat fraction (FF) in vertebral bone marrow using T2*-corrected, multipeak spectral modeling $(17,18)$. The fat fraction was obtained in each lumbar vertebra (L1 to L5); the average value over L1-L5 was calculated for each volunteer.

\section{Data Analysis}

The main data analysis was dedicated to the quantification of the diffusion and perfusion parameters in the lumbar vertebrae (L1 to L5) using the IVIM model. 
Regions-of-interest (ROIs) with an area of 21 voxels were drawn by a radiologist (XYZ, 18 years of experience) on the diffusion-weighted images acquired at b-value $=0 \mathrm{~s} / \mathrm{mm}^{2}$, in the vertebral bodies of $L 1$ to $L 5$; these ROls were then copied to the other images at different b-values. ROls were placed with care to avoid voxels with cortical bone, large blood vessels and partial volume effects.

The quantification of the diffusion coefficient $(D)$, perfusion fraction $(f)$ and pseudodiffusion coefficient $\left(D^{*}\right)$ was carried out by fitting the IVIM signal decay $(S)$ to the biexponential function $S\left(b_{n}\right)=S_{0}\left[f e^{-b_{n} D^{*}}+(1-f) e^{-b_{n} D}\right]$, where $b_{n}$ are the bvalues and $S_{0}$ is the fitted signal at b-value $=0 \mathrm{~s} / \mathrm{mm}^{2}$. To evaluate the effect of fat suppression on the quantification of IVIM parameters, the analysis was performed on the RESOLVE images acquired without fat suppression and on the three other RESOLVE acquisitions performed with fat suppression. The values of $D, f$ and $D^{*}$ were measured in each vertebra from L1 to L5; the average values over L1-L5 were then calculated for each volunteer. The bi-exponential data fitting was carried out using the nonlinear least-squares solver, as implemented by the Matlab function 'Isqcurvefit' (Matlab R2008, MathWorks, Natick, MA, USA).

Furthermore, an additional analysis was performed to assess the effect of the different fat suppression techniques on the signal intensity of subcutaneous fat, muscle and bone marrow on the RESOLVE images. Seven additional ROls were drawn on the images acquired at $b=0 \mathrm{~s} / \mathrm{mm}^{2}$ : one ROI in each of the five intervertebral discs located between the $\mathrm{L} 1$ and $\mathrm{S} 1$ vertebrae, one $\mathrm{ROI}$ in the periumbilical subcutaneous fat and one ROI in the paravertebral muscle. The signal of the subcutaneous fat, muscle and bone marrow was normalized using the signal in the intervertebral disc as a reference, since there is no signal originating from the lipids in this tissue. The normalized signal of subcutaneous fat, bone marrow and 
muscle was measured for the four different MRI datasets ( 3 with fat suppression and 1 without fat suppression).

To investigate the repeatability of IVIM-RESOLVE measurements with FS and SPAIR, six experiments were performed on a volunteer and two separate analyses were conducted.

In the first analysis, the characteristics of the signal obtained from FS and SPAIR were investigated. The signal of the IVIM diffusion decay of each measurement was normalized to the value of the signal acquired at $b=0 \mathrm{~s} / \mathrm{mm}^{2}$; then, the signals of six measurements were combined to provide a mean value, standard deviation and coefficient of variation (CV). The CV, which represents a measure of repeatability, was calculated for each lumbar vertebra. A more detailed description of this calculation is provided in the supplementary material (Table S1 and S2).

The second analysis was dedicated to the comparison between FS and SPAIR of the CV of $D, D^{*}$ and $f$. Preliminary analyses indicated that the signal at $\mathrm{b}=1000 \mathrm{~s} / \mathrm{mm}^{2}$ (which has the lowest signal-to-noise ratio of the decay curve) might exacerbate the CV of the IVIM parameters. Thus, $D, D^{*}$ and $f$ were determined in two ways: using six b-values, with the b-value $\max =800 \mathrm{~s} / \mathrm{mm}^{2}$ and using seven b-values, with the bvalue $_{\max }=1000 \mathrm{~s} / \mathrm{mm}^{2}$.

Overall, for each measurement a qualitative assessment of the images, with respect to signal-to-noise ratio, artifacts and fat suppression, was performed by radiologist (XYZ, 18 years of experience).

\section{Statistical Analysis}

The IVIM parameters $\left(D, f\right.$ and $\left.D^{*}\right)$ measured over all volunteers for each RESOLVE sequence (unsuppressed fat, FS, SPAIR and STIR acquisitions) were compared using a one-way repeated-measures analysis of variance (ANOVA) followed by 
Bonferroni's multiple comparison test. With respect to the repeatability measurements, the CV of the signal and the CV of the IVIM parameters obtained with FS and SPAIR were compared using a paired t-test. A threshold of $5 \%$ was chosen for statistical significance. Statistical analyses were performed with GraphPad Prism (GraphPad, La Jolla, CA, USA).

\section{RESULTS}

In Figure 1, an example of RESOLVE images (b-value $=0 \mathrm{~s} / \mathrm{mm}^{2}$ ) acquired with and without fat suppression is illustrated. The ROls used for data analysis are shown on the images. Qualitatively, a smaller signal-to-noise ratio is noticeable on the STIR image, when compared to the other images. In all four RESOLVE images, the very upper and lower regions of the image suffered from distortion artifacts. On the other hand, no artifacts were observed in the anatomical regions of interest for the quantification of the IVIM parameters in the vertebral bone marrow.

The MR images acquired with the FS, SPAIR and STIR modules showed a good and among them comparable - suppression of the subcutaneous fat. This observation was confirmed by the results of the semi-quantitative analysis shown in Table 2. The FS, SPAIR and STIR modules decreased the relative (i.e., normalized to the signal of the intervertebral disc) signal of the subcutaneous fat and bone marrow by a similar ratio. The relative signal of the muscle was little affected by the fat suppression. This analysis was performed on the RESOLVE images with $b$-value $=0$, 150 and $800 \mathrm{~s} / \mathrm{mm}^{2}$.

The quantification of the IVIM parameters, obtained from the bi-exponential fit of the diffusion signal decay acquired with/without fat suppression, is given in Figures 2, 3 and 4. 
The values of the diffusion coefficient in the vertebral bone marrow are shown in Figure 2. The diffusion coefficient was significantly lower in the acquisition without fat suppression than in the FS and SPAIR acquisitions $(p<0.01)$. There was no statistically significant difference among the values of $D$ obtained with FS, SPAIR and $\operatorname{STIR}(p=0.27)$.

Figure 3 illustrates the values of perfusion fraction obtained with and without fat suppression. The perfusion fraction was significantly lower in the acquisition without fat suppression than in the FS $(p<0.05)$ and STIR $(p<0.01)$ acquisitions. There was no statistically significant difference among the values of $f$ obtained with FS, SPAIR and $\operatorname{STIR}(p=0.16)$.

Table 3 shows the values of perfusion fraction measured for each volunteer, with and without fat suppression. The fat fraction for each volunteer is also provided. An intersubject variability of the perfusion fraction could be observed: $f$ ranged between $6 \pm 2$ $\%$ and $11 \pm 7 \%$, without fat suppression and between $9 \pm 3 \%$ and $16 \pm 2 \%$, with fat suppression. Similarly, a FF inter-subject variability was also found (18 $\pm 2 \%$ to $43 \pm$ $3 \%)$. Interestingly, the larger differences between the perfusion fractions obtained with fat suppression and that obtained without fat suppression were found in the volunteers (\#3, \#4) who displayed a high FF (31 $\pm 4 \%$ and $43 \pm 3 \%$, respectively).

The pseudo-diffusion coefficients (Figure 4) showed no significant differences between data acquired with and without fat suppression ( $p=0.36$, ANOVA).

The analysis of the repeatability measurements yielded a CV of the signal obtained with SPAIR significantly smaller than the CV with FS $(3.2 \pm 1.2 \%$ vs $4.2 \pm 2.2 \%, p=$ 0.002, paired t-test). With respect to the IVIM parameter quantification, SPAIR provided lower CVs for $D, D^{*}$ and $f$ compared to FS. Furthermore, the data analysis with b-value $\max =800 \mathrm{~s} / \mathrm{mm}^{2}$ (Table 4) yielded lower CVs compared to the analysis 
with b-value $\max =1000 \mathrm{~s} / \mathrm{mm}^{2}$ (Table S3, supplementary materials). When considering the analysis with b-value $e_{\max }=800 \mathrm{~s} / \mathrm{mm}^{2}$, the CV of $D$ obtained with SPAIR $(13.9 \pm 6.3 \%)$ was found to be smaller than the CV with FS $(21.2 \pm 3.5 \%)$; however this difference did not reach statistical significance $(p=0.08)$. A similar situation occurred for $D^{*}($ CV SPAIR $=46.7 \pm 16.6 \%$, CV FS $=60.3 \pm 8.7 \%, p=0.22)$ The CV of the perfusion fraction $f$ measured with SPAIR was significantly smaller than the CV obtained with FS $(47.6 \pm 17.3 \%$ vs $77.6 \pm 11.9 \%$, respectively, $\mathrm{p}=0.023)$

An example of the IVIM signal decay obtained in vertebral bone marrow with and without fat suppression is illustrated in Figure 5. The contribution of the fat to the total signal appears clearly on the signal decay without fat suppression. It is noteworthy that the initial slope, which is proportional to $f D^{*}$, is substantially smaller in the signal decay without fat suppression, when compared to the signal decay with fat suppression. This is in agreement with the results shown in Figure 3 and 4: the value of $\mathrm{f}$ is decreased in the acquisition without fat suppression and the value of $D^{*}$ is unchanged between the two acquisitions.

Given the significant effect of the lipid signal on the quantitation of the IVIM parameters, a novel IVIM model with three compartments and two chemical species (water and lipids) was proposed and illustrated in Figure 6. For instance, when we consider a voxel without adipocytes and with a fractional capillary blood volume of 0.2 , a perfusion fraction of $20 \%$ would be measured by the IVIM technique (Figure 6 ). In a voxel with adipocytes and a fractional capillary blood volume of 0.2 , the IVIM perfusion fraction measured with fat suppression will be different than $20 \%$ and will depend on the relative abundance of NMR-visible lipids in the voxel $(f=25 \%$ and $33 \%$, for a fractional lipid compartment of 0.2 and 0.4 , respectively, Figure 6 ). 


\section{DISCUSSION}

In the current study, we have implemented a spine IVIM protocol using the RESOLVE sequence. This protocol provided diagnostic-quality MR images in addition to the quantitative assessment of the IVIM parameters in vertebral bone marrow. Furthermore, the confounding effect of the lipid signal on the diffusion and perfusion quantification was assessed by applying different fat suppression modules. It was shown that the lipid signal contamination leads to an underestimation of both diffusion coefficient and perfusion fraction.

Despite the fact that quantitative MRI is well established in bone marrow $(19,20)$, only a limited number of studies have used the IVIM approach. An overview of the results from previous IVIM bone marrow studies can be presented in a simple, compact table (Table 5). These results span a relatively broad range of values: $D$ ranges between $0.20 \times 10^{-3} \mathrm{~mm}^{2} / \mathrm{s}$ and $0.88 \times 10^{-3} \mathrm{~mm}^{2} / \mathrm{s}, D^{*}$ between $24 \times 10^{-3} \mathrm{~mm}^{2} / \mathrm{s}$ and $67 \times 10^{-3} \mathrm{~mm}^{2} / \mathrm{s}$ and $f$ between $10 \%$ and $44 \%$. These differences might originate from differences among the cohorts (age, pathophysiology), data acquisition parameters, etc. It should be noted that all the published IVIM studies on bone marrow have been performed in very recent years (one in 2014, one in 2015, five in 2017); the increased interest in bone marrow IVIM is probably due to sequence and hardware improvements, which allow for a more reliable quantification of $D, D^{*}$ and $f$. With respect to the diffusion measurements in vertebral bone marrow, Dietrich et al. (10) presents an excellent review of the literature concerning the ADC values in healthy volunteers and patients. In previous studies, according to Dietrich et al. (10) the bone marrow $A D C$ measured with fat suppression at $1.5 \mathrm{~T}$ was found to range between $0.23 \times 10^{-3} \mathrm{~mm}^{2} / \mathrm{s}$ and $0.58 \times 10^{-3} \mathrm{~mm}^{2} / \mathrm{s}$, in healthy volunteers. This rather wide range of values could be again explained by differences among the studies in 
cohorts and MRI sequence parameters; furthermore, the fat suppression efficiency/ quality could also play a role. It should be pointed out that, in studies without fat suppression, the bone marrow ADC was found in the range of $0.21-0.42 \times 10^{-3} \mathrm{~mm}^{2} / \mathrm{s}$ (10).

In the current study we have investigated the effect of the lipid signal in the context of the IVIM measurements and two main findings are noteworthy. First, an underestimation of $D$ was observed in the measurements without fat suppression, when compared to the value of $D$ obtained with fat suppression. This result is in agreement with previous literature findings on water ADC measurements and can be explained by the fact that the lipid molecules are characterized by a slow Brownian motion, compared to water molecules (21). The underestimation of $D$ can also be evinced by visual inspection of the IVIM signal decay in Figure 5, where the slope for $\mathrm{b} \sim 100-800 \mathrm{~s} / \mathrm{mm}^{2}$ is less steep (i.e., smaller diffusion coefficient) in the absence of fat suppression.

The second finding relates to the effect of the lipid signal on the quantification of the perfusion fraction; this aspect has not been previously investigated in IVIM measurements. An underestimation of $f$ was observed in the measurements without fat suppression, when compared to the values obtained with fat suppression. Thus, these results indicate that the quality of fat suppression represents a critical aspect also in the quantification of the perfusion fraction $f$. However, the underestimation of $f$ caused by the presence of residual lipid signal is not the only issue originating from the presence of lipids in a given voxel. Indeed, even in the case of a 'perfect' fat suppression there is an important element that needs to be considered. In the IVIM model, as originally proposed by Le Bihan $(1,22)$, the basic assumption is that the DW-MR signal originates from one chemical species (water) composed of two 
compartments; the perfusion fraction $f$ is defined as "the fractional volume (\%) of capillary blood flowing in each voxel, or more exactly the ratio of the volume of NMRvisible water flowing in the capillary compartment [...] to the total voxel volume of NMR-visible water' (22). Thus, in the 'standard' IVIM model, any change in perfusion fraction -following therapy, for instance- can be directly attributed to a change in capillary blood volume.

A different situation occurs in bone marrow, which is a tissue with a non-negligible adipocyte content. It is important to point out that the changes (following an intervention, for instance) in bone marrow IVIM perfusion fraction should be interpreted with caution; moreover, it is advisable when performing IVIM in bone marrow to measure also the fat fraction, in order to assess whether perfusion fraction changes could be ascribed to changes in capillary blood volume or to bone marrow conversion/fatty-replacement effects. The additional fat fraction measurement does not represent a major constraint for the patient protocol, since nowadays these measurements can be obtained from a single breath-hold sequence; furthermore, the fat fraction maps are typically provided by the MRI vendors and are readily available on the MRI system console. It could be also useful to rename the IVIM perfusion fraction measured in bone marrow with fat suppression to $f_{F S}$, to highlight explicitly that i) the perfusion fraction is measured with fat suppression and ii) the fat fraction needs to be taken into account for the interpretation of the changes in $f_{F S}$. It should be also noted that, as in all other tissues, for an accurate IVIM measurement of the perfusion fraction the difference in relaxation times (T1 and T2) of tissue and blood should be taken into account.

In previous IVIM studies on vertebral bone marrow, single-shot EPI sequences have been employed (3-9). In the current study the use of a RESOLVE sequence -i.e., a 
segmented readout- allows for i) an increase of the matrix size, thus the acquisition of images with an improved spatial resolution and ii) the shortening of the EPI echo train, which contributes to reduce the distortions and blurring artifacts on the images. The disadvantage of the RESOLVE sequence, in comparison to the single-shot approach, is the longer acquisition time. On the other hand, the spine IVIM protocol proposed in the current study has an acquisition time of less than 5 min, thus it could be applied in clinical settings without major concerns. The analysis of the repeatability measurements, suggests that the SPAIR yields more precise measurements than the FS, in the context of diffusion-weighted RESOLVE imaging of the spine. However, more detailed studies are needed to confirm this finding.

The current study has a number of limitations. First, a small number of volunteers was examined. A study on a larger cohort will be necessary to 1) assess in more details the impact of FS and SPAIR modules on the quantification of $D, D^{*}$ and $f$ and 2) investigate the relationship between IVIM metrics and FF. Secondly, the data fitting was carried out using one fitting approach. An investigation of the different fitting methods, such as 'fixed D*', segmented (unconstrained/constrained), BayesianProbability, etc. (23), that have been recently applied to IVIM studies of other organs, will provide further insight into the quantification of $D, D^{*}$ and $f$ in bone marrow. In conclusion, IVIM-MRI measurements in vertebral bone marrow were performed with the RESOLVE sequence and the effect of lipids on the quantitation of the IVIM parameters was investigated. An underestimation of both $D$ and $f$ was observed in the measurements performed without fat suppression. Furthermore, the results of this study indicated that when measuring the IVIM parameters in bone marrow, it is advisable to measure the fat fraction, since changes in the measured perfusion 
fraction might originate either from changes in capillary blood volume or from changes in lipid content. 


\section{REFERENCES}

1. Le Bihan D, Breton E, Lallemand D, Grenier P, Cabanis E, Laval-Jeantet M. MR imaging of intravoxel incoherent motions: application to diffusion and perfusion in neurologic disorders. Radiology 1986;161:401-407.

2. Lemke A, Laun FB, Simon D, Stieltjes B, Schad, LR. An in vivo verification of the intravoxel incoherent motion effect in diffusion-weighted imaging of the abdomen. Magn Reson Med 2010;64:1580-1585.

3. Marchand AJ, Hitti E, Monge F, et al. MRI quantification of diffusion and perfusion in bone marrow by intravoxel incoherent motion (IVIM) and nonnegative least square (NNLS) analysis. Magn Reson Imaging 2014;32:1091-1096.

4. Bourillon C, Rahmouni A, Lin C et al. Intravoxel Incoherent Motion Diffusionweighted Imaging of Multiple Myeloma Lesions: Correlation with Whole-Body Dynamic Contrast Agent-enhanced MR Imaging. Radiology 2015;277:773-783.

5. Baik JS, Jung, JY, Jee WH et al. Differentiation of focal indeterminate marrow abnormalities with multiparametric MRI. J Magn Reson Imaging 2017;46:49-60.

6. Lee EYP, Perucho JAU, Vardhanabhuti $V$ et al. Intravoxel incoherent motion MRI assessment of chemoradiation-induced pelvic bone marrow changes in cervical cancer and correlation with hematological toxicity. J Magn Reson Imaging 2017;46:1491-1498. 
7. Niu J, Li W, Wang $\mathrm{H}$ et al. Intravoxel incoherent motion diffusion-weighted imaging of bone marrow in patients with acute myeloid leukemia: a pilot study of prognostic value. J Magn Reson Imaging 2017;46:476-482.

8. Park S, Kwack KS, Chung NS, et al. Intravoxel incoherent motion diffusionweighted magnetic resonance imaging of focal vertebral bone marrow lesions: initial experience of the differentiation of nodular hyperplastic hematopoietic bone marrow from malignant lesions. Skeletal Radiol 2017;46:675-683.

9. Yoon MA, Hong SJ, Lee $\mathrm{CH}$, Kang $\mathrm{CH}$, Ahn KS, Kim BH. Intravoxel incoherent motion (IVIM) analysis of vertebral bone marrow changes after radiation exposure from diagnostic imaging and interventional procedures. Acta Radiol 2017;58:1260-1268.

10.Dietrich O, Geith T, Reiser MF, Baur-Melnyk A. Diffusion imaging of the vertebral bone marrow. NMR Biomed 2017;30:i-i. doi:10.1002/nbm.3333

11. Biffar A, Sourbron S, Schmidt $G$ et al. Measurement of perfusion and permeability from dynamic contrast-enhanced MRI in normal and pathological vertebral bone marrow. Magn Reson Med 2010;64:115-124.

12. Dieckmeyer M, Ruschke S, Eggers $H$ et al. ADC Quantification of the Vertebral Bone Marrow Water Component: Removing the Confounding Effect of Residual Fat. Magn Reson Med 2017;78:1432-1441.

13. Porter DA and Heidemann RM. High resolution diffusion-weighted imaging using readout-segmented echo-planar imaging, parallel imaging and a twodimensional navigator-based reacquisition. Magn Reson Med 2009;62:468-475. 
14. Hayes LL, Jones RA, Palasis S, Aguilera D, Porter DA. Drop metastases to the pediatric spine revealed with diffusion-weighted MR imaging. Pediatr Radiol 2012;42:1009-1013.

15. Kim TH, Baek MY, Park JE et al. Comparison of DWI Methods in the Pediatric Brain: PROPELLER Turbo Spin-Echo Imaging Versus Readout-Segmented Echo-Planar Imaging Versus Single-Shot Echo-Planar Imaging. AJR Am J Roentgenol 2018;210:1-7.

16. Klauser AS, Ellah MA, Kremser C, et al. Carpal tunnel syndrome assessment with diffusion tensor imaging: value of fractional anisotropy and apparent diffusion coefficient. Eur Radiol, 2018;28:1111-1117.

17.Zhong X, Nickel MD, Kannengiesser SA, Dale BM, Kiefer B, Bashir MR. Liver fat quantification using a multi-step adaptive fitting approach with multi-echo GRE imaging. Magn Reson Med 2014;72:1353-1365.

18. Le Ster C, Gambarota G, Lasbleiz J, Guillin R, Decaux O, Saint-Jalmes H. Breath-hold MR measurements of fat fraction, $\mathrm{T} 1$, and $\mathrm{T}^{*}$ of water and fat in vertebral bone marrow. J Magn Reson Imaging 2016;44:549-555.

19. Karampinos DC, Ruschke S, Dieckmeyer M et al. Quantitative MRI and spectroscopy of bone marrow. Magn Reson Imaging 2018;47:332-353.

20.Singhal V, Bredella MA. Marrow adipose tissue imaging in humans. Bone 2018 doi: 10.1016/j.bone.2018.01.009. [Epub ahead of print]

21.Lehnert A, Machann J, Helms G, Claussen CD, Schick F. Diffusion characteristics of large molecules assessed by proton MRS on a whole-body MR system. Magn Reson Imaging 2004;22:39-46.

22. Le Bihan D and Turner R. The capillary network: a link between IVIM and classical perfusion. Magn Reson Med 1992;27:171-178. 
23. Barbieri S, Donati OF, Froehlich, JM, Thoeny, HC. Impact of the calculation algorithm on biexponential fitting of diffusion-weighted MRI in upper abdominal organs. Magn Reson Med 2016;75 :2175-2184. 
Table 1

RESOLVE sequence parameters of the spine IVIM protocol.

\begin{tabular}{lc}
\hline & RESOLVE IVIM \\
\hline TR/TE (ms) & $2400 / 58$ \\
Matrix & $188 \times 188$ \\
Slices & 10 \\
Slice thickness (mm) & 6 \\
BW (Hz/px) & 1330 \\
Orientation & Sagittal \\
FOV (mm) & $400 \times 400$ \\
iPAT & 3 \\
Segments in readout direction & 3 \\
Scan Time (min:sec) & $4: 41$ \\
Number of Averages & 2 \\
b-values (s/mm ${ }^{2}$ ) & $0,50,100,150,400,800,1000$ \\
\hline
\end{tabular}




\section{Table 2}

The normalized signal of subcutaneous fat, paravertebral muscle and bone marrow, measured without fat suppression (No Fat Sup) and with different fat suppression techniques $(\mathrm{FS}=$ Fat Saturation, SPAIR = Spectral Attenuated Inversion Recovery, STIR = Short Tau Inversion Recovery). Similar suppression of the fat signal was observed with the three fat suppression techniques, at different b-values.

\begin{tabular}{|c|c|c|c|c|c|}
\hline \multirow{2}{*}{$\begin{array}{c}\text { b-value (s/ } \\
\mathrm{mm}^{2} \text { ) }\end{array}$} & \multirow[t]{2}{*}{ ROI } & \multicolumn{4}{|c|}{ Normalized Signal } \\
\hline & & $\begin{array}{l}\text { No Fat } \\
\text { Sup }\end{array}$ & FS & SPAIR & STIR \\
\hline \multirow[t]{4}{*}{0} & Subcutaneous & 0.67 & 0.14 & 0.14 & 0.17 \\
\hline & Fat & & & & \\
\hline & Muscle & 0.29 & 0.27 & 0.25 & 0.26 \\
\hline & Bone Marrow & 0.68 & 0.42 & 0.44 & 0.45 \\
\hline \multirow[t]{4}{*}{150} & Subcutaneous & 0.80 & 0.19 & 0.19 & 0.19 \\
\hline & Fat & & & & \\
\hline & Muscle & 0.29 & 0.27 & 0.24 & 0.26 \\
\hline & Bone Marrow & 0.84 & 0.48 & 0.50 & 0.52 \\
\hline \multirow[t]{4}{*}{800} & Subcutaneous & 2.36 & 0.36 & 0.38 & 0.39 \\
\hline & Fat & & & & \\
\hline & Muscle & 0.38 & 0.31 & 0.29 & 0.38 \\
\hline & Bone Marrow & 2.46 & 1.24 & 1.34 & 1.37 \\
\hline
\end{tabular}




\section{Table 3}

The value of the perfusion fraction in each volunteer, assessed without fat suppression (no Fat Sup) and with fat suppression (SPAIR), averaged over L1-L5. The fat fraction (FF) in each volunteer is also displayed.

\begin{tabular}{lcccccc}
\hline \multicolumn{1}{c}{ Volunteer } & $\# 1$ & $\# 2$ & $\# 3$ & $\# 4$ & $\# 5$ & $\# 6$ \\
\hline $\mathrm{f}(\%)$ no Fat Sup & $7 \pm 3$ & $6 \pm 3$ & $7 \pm 2$ & $8 \pm 2$ & $11 \pm 7$ & $6 \pm 2$ \\
$\mathrm{f}(\%)$ SPAIR & $12 \pm 5$ & $9 \pm 3$ & $16 \pm 2$ & $15 \pm 2$ & $12 \pm 6$ & $11 \pm 2$ \\
$\mathrm{FF}(\%)$ & $34 \pm 6$ & $18 \pm 2$ & $31 \pm 4$ & $43 \pm 3$ & $26 \pm 4$ & $20 \pm 2$ \\
\hline
\end{tabular}


Table 4. Results of the repeatability study $\left(b-v^{2}\right.$ lue $\left.\max =800 \mathrm{~s} / \mathrm{mm}^{2}\right)$. The mean values and standard deviations of $D, D^{*}$ and $f$ are calculated over six measurements, for the vertebrae L1 to L5. The last row provides the mean value and standard deviation calculated over the five vertebrae.

\begin{tabular}{cccc|ccc} 
& \multicolumn{3}{c}{ SPAIR } & \multicolumn{3}{c}{ FS } \\
\cline { 2 - 7 } & $\mathrm{D}$ & $\mathrm{D}^{*}$ & $\mathrm{f}$ & $\mathrm{D}$ & $\mathrm{D}^{*}$ & $\mathrm{f}$ \\
& $\left(10^{-3} \mathrm{~mm}^{2} /\right.$ & $\left(10^{-3} \mathrm{~mm}^{2} /\right.$ & $(\%)$ & $\left(10^{-3} \mathrm{~mm}^{2} /\right.$ & $\left(10^{-3} \mathrm{~mm}^{2 /}\right.$ & $(\%)$ \\
$\mathrm{s})$ & $\mathrm{s})$ & & $\mathrm{s})$ & $\mathrm{s})$ & \\
\hline $\mathrm{L} 1$ & $0.44 \pm$ & $10.6 \pm 4.9$ & $10.3 \pm 8.0$ & $0.47 \pm$ & $15.0 \pm$ & $10.0 \pm 7.9$ \\
& 0.10 & & & 0.10 & 11.1 & \\
\hline L2 & $0.49 \pm$ & $10.9 \pm 5.8$ & $9.2 \pm 3.4$ & $0.47 \pm$ & $6.4 \pm 3.3$ & $13.8 \pm 8.1$ \\
& 0.03 & & & 0.09 & & \\
\hline L3 & $0.49 \pm$ & $14.2 \pm 9.5$ & $9.7 \pm 3.3$ & $0.49 \pm$ & $19.9 \pm$ & $13.2 \pm$ \\
& 0.08 & & & 0.11 & 11.7 & 10.5 \\
\hline L4 & $0.52 \pm$ & $17.2 \pm 8.0$ & $7.6 \pm 3.3$ & $0.46 \pm$ & $16.5 \pm 9.0$ & $11.0 \pm$ \\
& 0.07 & & & 0.07 & & 10.1 \\
\hline L5 & $0.45 \pm$ & $15.4 \pm 3.2$ & $7.9 \pm 3.7$ & $0.38 \pm$ & $10.2 \pm 6.4$ & $10.9 \pm 8.6$ \\
& 0.05 & & & 0.10 & & \\
\hline Mean \pm & $0.48 \pm$ & $13.7 \pm 2.9$ & $8.9 \pm 1.2$ & $0.45 \pm$ & $13.6 \pm 5.3$ & $11.8 \pm 1.6$ \\
SD & 0.04 & & & 0.04 & &
\end{tabular}




\section{Table 5}

The values of the diffusion coefficient $(D)$, the pseudo-diffusion coefficient $\left(D^{*}\right)$ and the perfusion fraction $(f)$ in vertebral bone marrow (with exception of [5]), measured in the current and previous IVIM studies.

\begin{tabular}{|c|c|c|c|c|}
\hline & & $\begin{array}{c}D \\
\left(\times 10^{-3} \mathrm{~mm}^{2} /\right. \\
\mathrm{s})\end{array}$ & $\begin{array}{c}D^{*} \\
\left(\times 10^{-3} \mathrm{~mm}^{2} /\right. \\
\mathrm{s})\end{array}$ & $\begin{array}{c}f \\
(\%)\end{array}$ \\
\hline Current study a & Healthy volunteers & $0.42 \pm 0.06$ & $16 \pm 6$ & $12 \pm 3$ \\
\hline [2] Marchand et al. ${ }^{b}$ & Healthy volunteers & $0.60 \pm 0.09$ & $28 \pm 9$ & $14 \pm 6$ \\
\hline \multirow[t]{2}{*}{ [3] Bourillon et al.c } & Focal-lesion free & $0.52 \pm 0.18$ & $26 \pm 19$ & $9 \pm 4$ \\
\hline & Focal lesions & $0.76 \pm 0.18$ & $37 \pm 34$ & $11 \pm 4$ \\
\hline \multirow[t]{2}{*}{ [4] Baïk et al.d } & Benign lesions & 0.41 & 0.26 & 11 \\
\hline & Malignant lesions & 0.92 & 0.27 & 13 \\
\hline [5] Lee et al.e & Pelvic bone marrow & $0.29 \pm 0.05$ & - & $44 \pm 4$ \\
\hline [6] Niu et al. ${ }^{f}$ & $\begin{array}{l}\text { Acute myeloid } \\
\text { leukemia }\end{array}$ & $0.24 \pm 0.04$ & $67 \pm 7$ & $22 \pm 5$ \\
\hline \multirow[t]{2}{*}{ [7] Park et al.g } & Nodular lesions & $0.22 \pm 0.08$ & $37 \pm 34$ & $20 \pm 9$ \\
\hline & Malignant lesions & $0.79 \pm 0.29$ & $32 \pm 31$ & $10 \pm 5$ \\
\hline [8] Yoon et al.h & $\begin{array}{l}\text { He p a t o c e I l u la r } \\
\text { carcinoma }\end{array}$ & 0.36 & 24 & 11 \\
\hline
\end{tabular}

a Values obtained with the SPAIR fat suppression.

b Cohort of fifteen women (aged between 18 and 30 years); STIR fat suppression.

c Patients with multiple myeloma; values obtained before treatment. Focal-lesion free tissue might consist of bone marrow with diffuse involvement or bone marrow without tumor infiltration.

d Patients with focal marrow abnormalities in different anatomical locations (femur, vertebra, etc.). In this study, there is probably a typo in the reporting of $D^{*}$ values since $D^{*}$ cannot be smaller than $D$. Results of Reader 1.

e Patients with cervical cancer; values obtained before chemoradiation treatment. Measurements were performed on pelvic bone marrow; $D^{*}$ data were not provided.

f Patients with acute myeloid leukemia before complete remission.

g Patients with focal vertebral bone marrow lesions. Results of Reader 1.

$\mathrm{h}$ Values obtained in vertebral bone marrow of patients with hepatocellular carcinoma, before radiation exposure. 


\section{Figure Legends}

Figure 1. Sagittal RESOLVE MR images of the spine of a 23-year-old woman. From the left to the right, images acquired: with Short Tau Inversion Recovery (STIR), with Spectral Attenuated Inversion Recovery (SPAIR), with Fat Saturation (FS) and without fat suppression. The regions-of-interest used for the data analysis are also displayed.

Figure 2. The value of the diffusion coefficient $(D)$ in vertebral bone marrow, obtained from the IVIM measurements and averaged over all volunteers. The value of $D$ obtained without fat suppression is smaller than that with fat suppression, due to the contamination of the lipid signal on the total signal.

Figure 3. The value of the perfusion fraction $(f)$ in vertebral bone marrow, obtained from the IVIM measurements and averaged over all volunteers. Similarly to the diffusion coefficient, without fat suppression the perfusion fraction is significantly smaller than with fat suppression.

Figure 4. The value of the pseudo-diffusion coefficient $D^{*}$ in vertebral bone marrow, obtained from the IVIM measurements and averaged over all volunteers. No effect of the fat suppression is observed on the $D^{*}$ value.

Figure 5. An example of two IVIM diffusion decay curves obtained in vertebral bone marrow, one acquired with fat suppression (blue, continuous line) and the other acquired without fat suppression (red, dot-line). Without fat suppression, the signal of 
lipid protons contributes to total signal, leading to inaccurate quantification of the diffusion coefficient and perfusion fraction.

Figure 6. An illustration of the IVIM model -as originally proposed by Le Bihan- and the IVIM model in bone marrow. The blood capillary compartment is represented in red, the tissue water compartment in orange and the lipid compartment in grey. In all three cases, a fractional capillary blood volume of 0.2 is considered in a given voxel. It can be observed that: i) the IVIM model in bone marrow would consist of three compartments and two chemical species (water and lipids), since the signal of lipids is not negligible with respect to the water signal and ii) in an IVIM measurement with fat suppression, the same fraction of capillary blood volume in a given voxel could yield different values of the perfusion fraction $f_{F S}$, depending on the voxel fat content. 

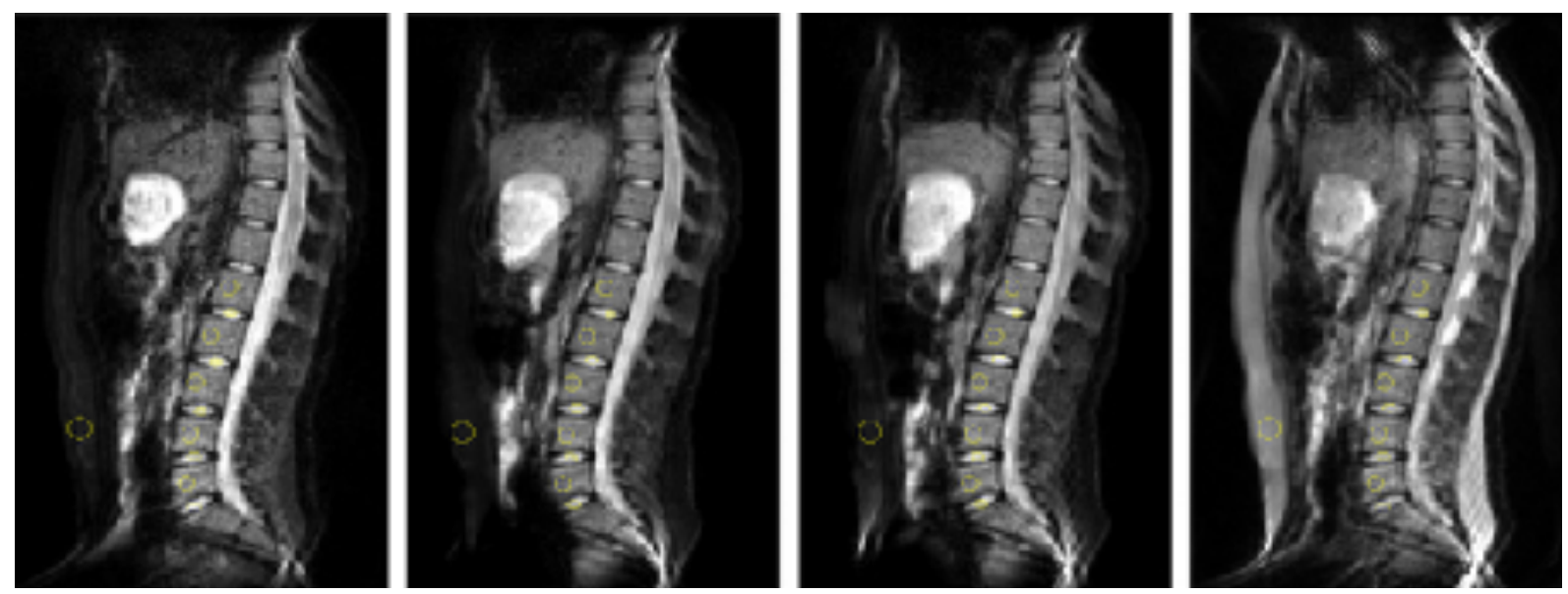

Figure 1. Sagittal RESOLVE MR images of the spine of a 23-year-old woman. From the left to the right, images acquired: with Short Tau Inversion Recovery (STIR), with Spectral Attenuated Inversion Recovery (SPAIR), with Fat Saturation (FS) and without fat suppression. The regions-of-interest used for the data analysis are also displayed 


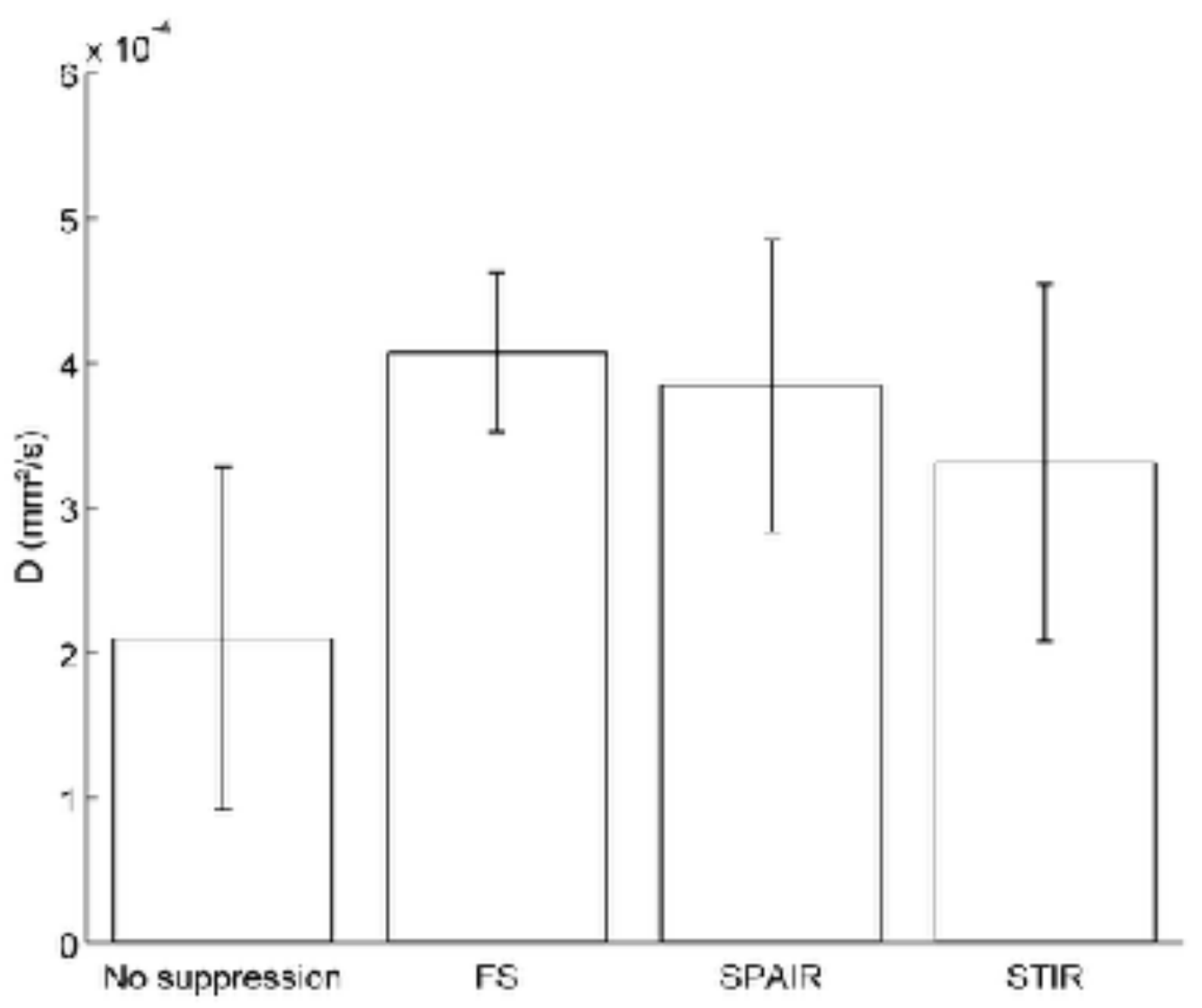

Figure 2. The value of the diffusion coefficient $(D)$ in vertebral bone marrow, obtained from the IVIM measurements and averaged over all volunteers. The value of $D$ obtained without fat suppression is smaller than that with fat suppression, due to the contamination of the lipid signal on the total signal. 


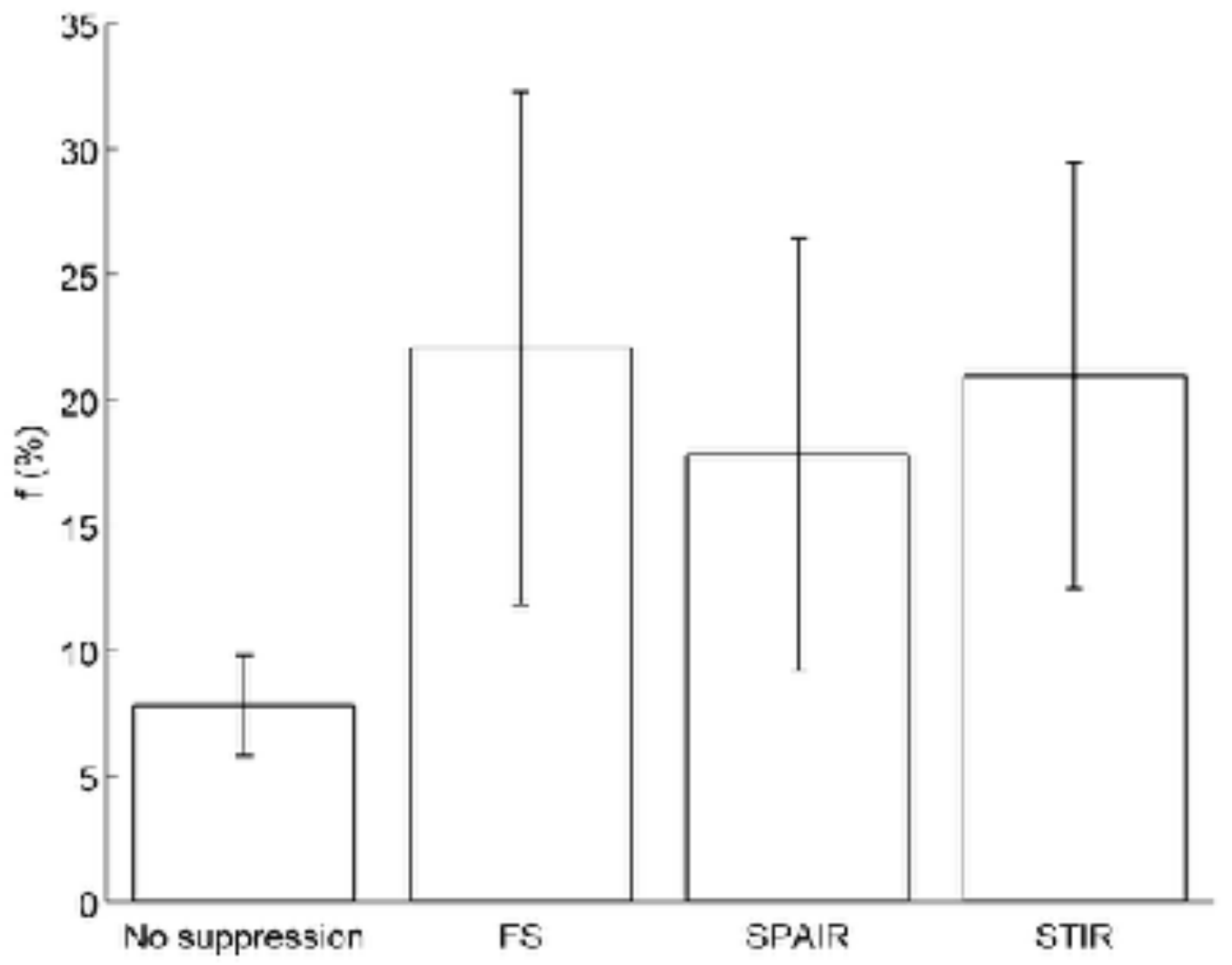

Figure 3. The value of the perfusion fraction $(f)$ in vertebral bone marrow, obtained from the IVIM measurements and averaged over all volunteers. Similarly to the diffusion coefficient, without fat suppression the perfusion fraction is significantly smaller than with fat suppression. 


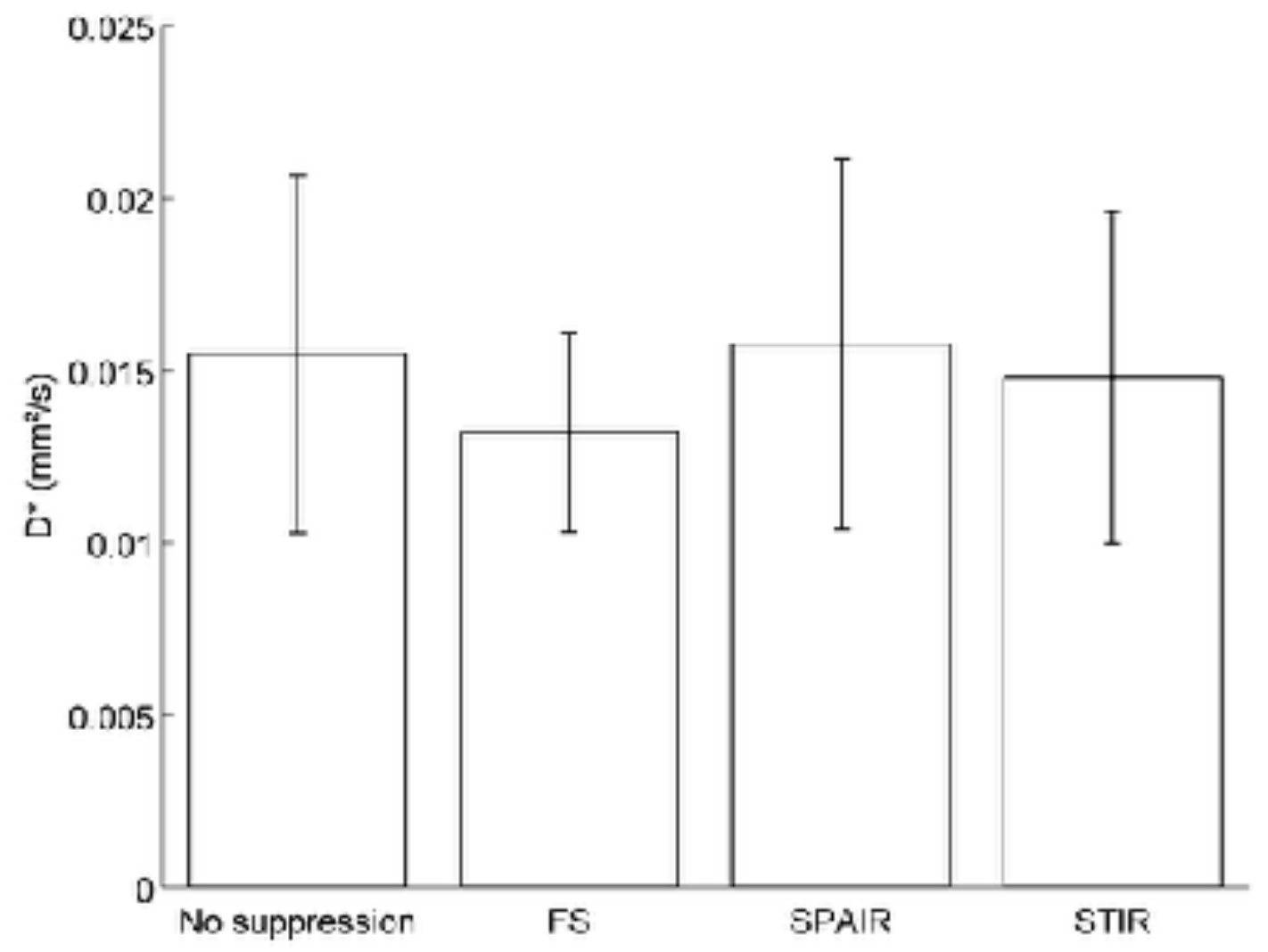

Figure 4. The value of the pseudo-diffusion coefficient $D^{*}$ in vertebral bone marrow, obtained from the IVIM measurements and averaged over all volunteers. No effect of the fat suppression is observed on the $D^{*}$ value. 


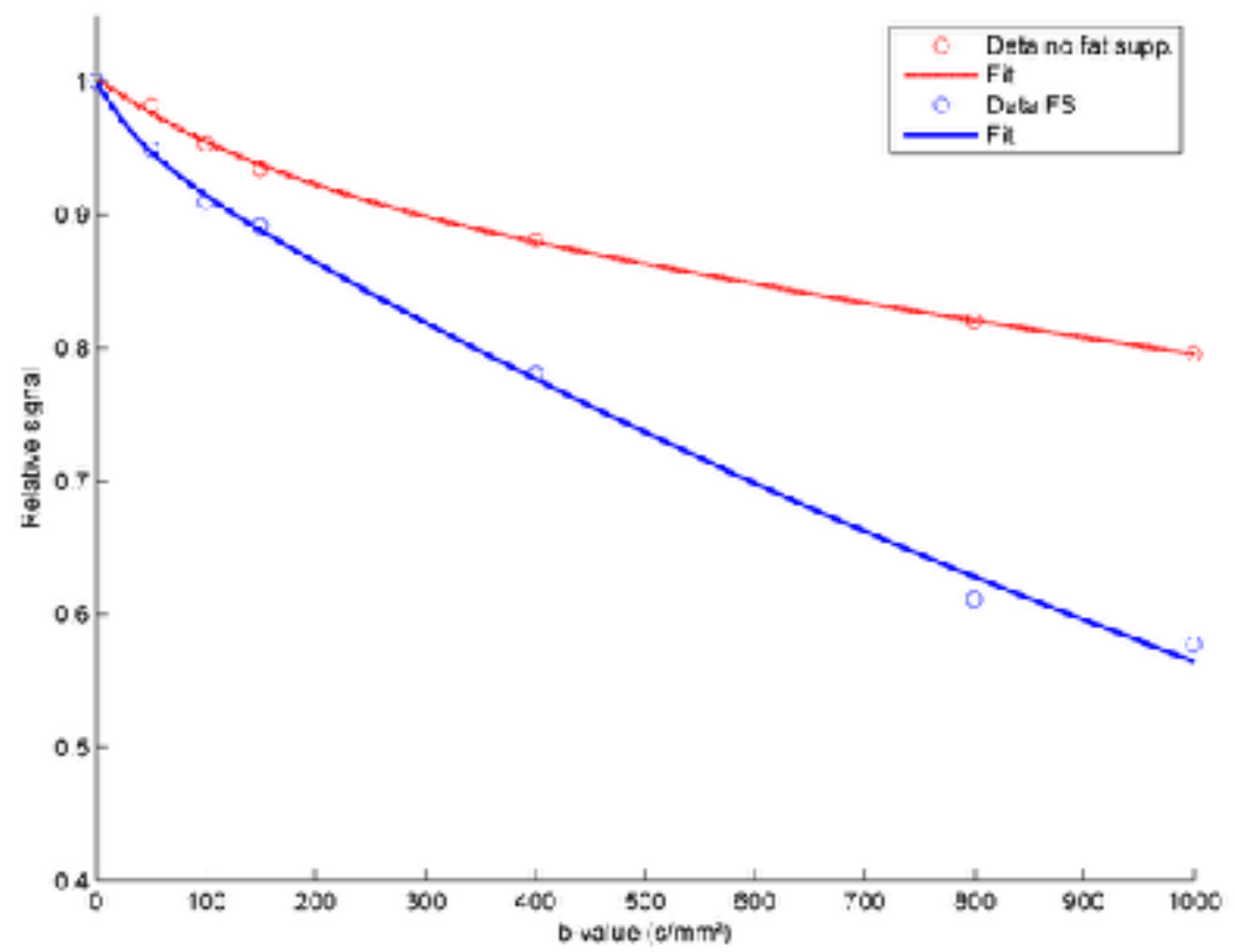

Figure 5. An example of two IVIM diffusion decay curves obtained in vertebral bone marrow, one acquired with fat suppression (blue, continuous line) and the other acquired without fat suppression (red, dot-line). Without fat suppression, the signal of lipid protons contributes to total signal, leading to inaccurate quantification of the diffusion coefficient and perfusion fraction. 
IVIM model

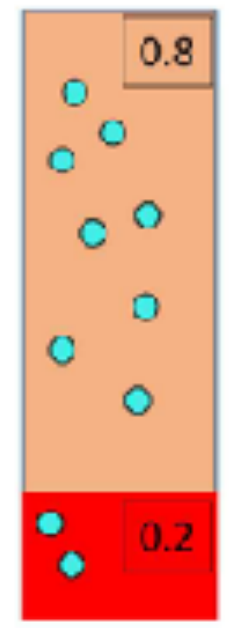

$f=20 \%$

\section{IVIM model in bone marrow}
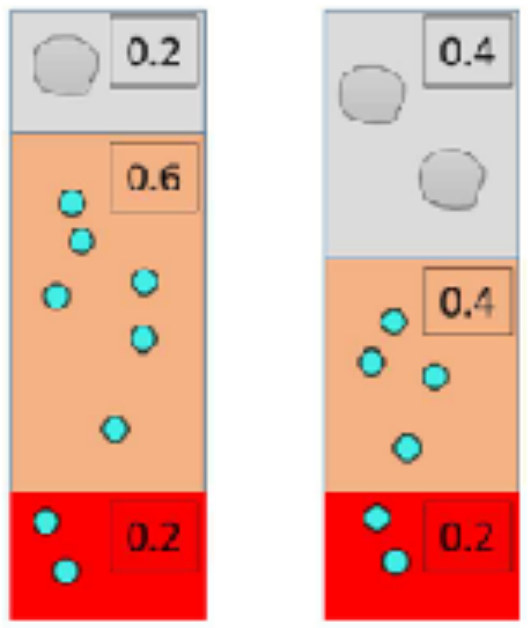

$f_{F S}=25 \% \quad f_{F 5}=33 \%$
- water

lipids

Figure 6. An illustration of the IVIM model -as originally proposed by Le Bihan- and the IVIM model in bone marrow. The blood capillary compartment is represented in red, the tissue water compartment in orange and the lipid compartment in grey. In all three cases, a fractional capillary blood volume of 0.2 is considered in a given voxel. It can be observed that: i) the IVIM model in bone marrow would consist of three compartments and two chemical species (water and lipids), since the signal of lipids is not negligible with respect to the water signal and ii) in an IVIM measurement with fat suppression, the same fraction of capillary blood volume in a given voxel could yield different values of the perfusion fraction $f_{F S}$, depending on the voxel fat content. 